PROCEEDINGS OF THE

AMERICAN MATHEMATICAL SOCIETY

Volume 136, Number 6, June 2008, Pages 1967-1975

S 0002-9939(08)09224-1

Article electronically published on February 15, 2008

\title{
NON-VANISHING OF THE TWISTED COHOMOLOGY ON THE COMPLEMENT OF HYPERSURFACES
}

\author{
YUKIHITO KAWAHARA
}

(Communicated by Ted Chinburg)

\begin{abstract}
Generically, the cohomology with coefficients in a local system of rank one on the complement in $\mathbb{P}^{n}$ of the union of a finite number of hypersurfaces vanishes except in the highest dimension. We study the non-generic case, in which the cohomology in other dimensions does not vanish. When the hypersurfaces are hyperplanes, many examples of this kind are known. In this paper, we consider the case in which the hypersurfaces need not be hyperplanes. We prove that the hypersurfaces given by some particular linear systems have non-vanishing local system cohomologies.
\end{abstract}

\section{INTRODUCTION}

Let $V_{1}, \ldots, V_{m}$ be hypersurfaces in the complex projective space $\mathbb{P}^{n}$ of dimension $n$ and let $\mathcal{L}$ be a complex local system of rank one over the complement $M=\mathbb{P}^{n} \backslash \bigcup_{i=1}^{m} V_{i}$. Call $H^{k}(M, \mathcal{L})$ the $k^{t h}$ twisted cohomology group of $M$. In [A, KN, Ch, Di] it is shown that if $V_{1}, \ldots, V_{m}$ and $\mathcal{L}$ are generic,

$$
H^{k}(M, \mathcal{L})=0 \quad \text { for } k \neq n .
$$

In particular, vanishing theorems for the case of hyperplanes were found in $\mathrm{Ko}$, Yu, CDO, Ka2 (cf. [ESV, STV]). Recently, arrangements of hyperplanes with non-vanishing twisted cohomology were studied and many examples were found (cf. [CS, Fa, LY, Ka3]). The results in $\mathrm{Yu2}$ imply that in $\mathbb{P}^{2}$, most examples of this kind consist of special elements of pencils. In this paper we generarize this result to hypersurfaces of arbitrary dimension. We shall show that there are hypersurfaces which are supports of divisors in some linear system with the property that their complements have non-vanishing twisted cohomologies in other than the top dimension. See Li2 for background and a discussion of related topics.

Let $\Omega_{M}$ denote the sheaf of germs of holomorphic forms on $M$ and let $\mathcal{O}_{M}=\Omega_{M}^{0}$. Let $D_{1}, \ldots, D_{m}$ be effective divisors on $\mathbb{P}^{n}$ such that the support of $D_{i}$ is $V_{i}$ and $D_{i}$ and $D_{j}$ are linearly equivalent for $i \neq j$. In this case, the $D_{i}$ 's have the same degree (see [H] $)$. Let $\lambda=\left(\lambda_{1}, \ldots, \lambda_{m}\right)$ be a complex weight with $\sum_{i=1}^{m} \lambda_{i}=0$. For $i \neq j, D_{i}-D_{j}$ is the divisor of some rational function $f_{i j}$. Fix $j$ and define the

Received by the editors February 26, 2006 and, in revised form, May 7, 2007.

2000 Mathematics Subject Classification. Primary 14F40; Secondary 14C20, 32S22.

Key words and phrases. Local system, twisted cohomology, linear system, hypersurface complement, hyperplane arrangement.

(C)2008 American Mathematical Society Reverts to public domain 28 years from publication 
global one-form $\omega_{\lambda}=\sum_{i \neq j} \lambda_{i} \mathrm{~d} \log f_{i j}$. It is independent of the choice of $j$ and we can denote

$$
\omega_{\lambda}=\sum_{i=1}^{m} \lambda_{i} \mathrm{~d} \log D_{i} \in \Gamma\left(M, \Omega_{M}^{1}\right), \quad \sum_{i=1}^{m} \lambda_{i}=0 .
$$

Define the flat connection $\nabla_{\lambda}=d+\omega_{\lambda} \bigwedge: \mathcal{O}_{M} \rightarrow \Omega_{M}^{1}$ and let the local system $\mathcal{L}_{\lambda}$ be the kernel of $\nabla_{\lambda}$. Since $M$ is a Stein manifold, we have

$$
H^{k}\left(M, \mathcal{L}_{\lambda}\right) \simeq H^{k}\left(\Gamma\left(M, \Omega_{M}\right), \nabla_{\lambda}\right)
$$

and $H^{k}\left(M, \mathcal{L}_{\lambda}\right)=0$ for $k>n$ (cf. [De]). The main result of this paper is the following:

Theorem 1. Let $1<n<s \leq m$. Let $D_{1}, \ldots, D_{m}$ be effective divisors on $\mathbb{P}^{n}$ with the same degree and let $M$ be the complement of their supports. Suppose

(A1) $D_{1}, \ldots, D_{s}$ are elements of some linear system $\Lambda$ on $\mathbb{P}^{n}$ with dimension $n-1$.

(A2) There exists a base point $P$ of $\Lambda$ such that $D_{1}, \ldots, D_{n}$ has local normal crossings at $P$ and none of the $D_{i}$ pass through $P$ for $i=s+1, \ldots, m$.

(A3) $D_{1}, \ldots, D_{s}$ are in general position as points in $\Lambda=\mathbb{P}^{n-1}$, namely, no $n$ of them lie on a hyperplane.

Let $\lambda \notin \mathbb{Z}^{m}$ be a non-trivial weight such that $\sum_{i=1}^{s} \lambda_{i}=0$ and $\lambda_{i}=0$ for $i=$ $s+1, \ldots, m$. Then we have

$$
\operatorname{dim} H^{n-1}\left(M, \mathcal{L}_{\lambda}\right) \geq\left(\begin{array}{c}
s-2 \\
n-1
\end{array}\right)
$$

Moreover, if $s<m$, then we have

$$
\operatorname{dim} H^{n}\left(M, \mathcal{L}_{\lambda}\right) \geq\left(\begin{array}{l}
s-2 \\
n-1
\end{array}\right) .
$$

Remark. The support of $\sum_{i=1}^{s} D_{i}$ is of great interest when considering the nonvanishing of twisted cohomology. Condition (A2) implies that, locally at $P$, the set $\left\{D_{1, P}, \ldots, D_{s, P}\right\}$ becomes a generic arrangement of $s$ hyperplanes.

Remark. If the support of $\sum_{i=1}^{m} D_{i}$ contains a hyperplane $H$, we can consider $M$ as the complement of affine hypersurfaces in $\mathbb{C}^{n}=\mathbb{P}^{n} \backslash H$. The case of affine hypersurfaces case was considered in [KN, Ki] (cf. Section [5).

Remark. Let $D_{1}, \ldots, D_{m}$ be divisors of degree $d$ defined only by hyperplanes. Let $\mathcal{A}$ be the set of irreducible components of the support of $\sum_{i=1}^{m} D_{i}$. Then $\mathcal{A}$ is an arrangement of hyperplanes in $\mathbb{P}^{n}$ and $M=\mathbb{P}^{n} \backslash \bigcup_{H \in \mathcal{A}} H$.

If $D_{1}, \ldots, D_{m}$ are divisors of degree one, then they are defined by hyperplanes. In this case, $\left\{D_{1}, \ldots, D_{s}\right\}$ becomes a generic arrangement of hyperplanes with center $P=\bigcap_{i=1}^{s} D_{i}$, and $D_{s+1}, \ldots, D_{m}$ are hyperplanes not passing through $P$. Theorem 1 in this case is well-known (cf. [Fa]).

In order to prove Theorem 1, we shall construct explicit non-trivial twisted cohomology classes (Section 3) using a natural generalization of the Arnold-OrlikSolomon relation (Section 2). Condition (A3) is not essential, and in Section 4 we generalize the theorem. In Section 6 we give examples. 


\section{Preliminary: Rational Forms}

Let $V$ be a vector space of dimension $\ell$ over a field $K$ of characteristic zero. Let $S=K[V]$ be the symmetric algebra of $V^{*}$ and let $F=K(V)$ be the quotient field of $S$. We consider $S$ as the polynomial algebra and $F$ as the field of rational functions on $V$. Let $\Omega(V)=\bigoplus_{p=0}^{\ell} \Omega^{p}(V)$ be the exterior algebra of the $F$-vector space $F \otimes V^{*}$ and let $d$ be the usual differential. When we choose a basis $x_{1}, \ldots, x_{\ell}$ of $V^{*}$, we have $S=K\left[x_{1}, \ldots, x_{\ell}\right], F=K\left(x_{1}, \ldots, x_{\ell}\right)$,

$$
\mathrm{d} f=\sum_{i=1}^{\ell} \frac{\partial f}{\partial x_{i}} \otimes x_{i}=\sum_{i=1}^{\ell} \frac{\partial f}{\partial x_{i}} \mathrm{~d} x_{i} \quad \text { for } f \in F,
$$

$\Omega^{0}(V)=F, \Omega^{1}(V)=F \otimes V^{*}=F \mathrm{~d} x_{1} \oplus \cdots \oplus F \mathrm{~d} x_{\ell}$, and,$\Omega^{p}(V)=\bigoplus_{i_{1}<\cdots<i_{p}} F \mathrm{~d} x_{i_{1}}$ $\wedge \cdots \wedge \mathrm{d} x_{i_{p}}$. For $p \geq 2$ and $\omega_{1}, \ldots, \omega_{p} \in \Omega^{1}(V)$, define

$$
\Delta\left[\omega_{1}: \cdots: \omega_{p}\right]:=\sum_{k=1}^{p}(-1)^{k-1} \omega_{1} \wedge \cdots \wedge \widehat{\omega_{k}} \wedge \cdots \wedge \omega_{p} .
$$

Lemma 2. Let $p \geq 2$ and $\omega_{1}, \ldots, \omega_{p} \in \Omega^{1}(V)$.

(1) For a permutation $\sigma$ of $\{1, \ldots, p\}$, we have

$$
\Delta\left[\omega_{\sigma(1)}: \cdots: \omega_{\sigma(p)}\right]=\operatorname{sign}(\sigma) \Delta\left[\omega_{1}: \cdots: \omega_{p}\right] .
$$

(2) If $2 \leq j \leq p-2$, then

$$
\begin{aligned}
\Delta\left[\omega_{1}: \cdots: \omega_{p}\right]= & \Delta\left[\omega_{1}: \cdots: \omega_{j}\right] \wedge \omega_{j+1} \wedge \cdots \wedge \omega_{p} \\
& +(-1)^{j} \omega_{1} \wedge \cdots \wedge \omega_{j} \wedge \Delta\left[\omega_{j+1}: \cdots: \omega_{p}\right] .
\end{aligned}
$$

(3) $\Delta\left[\omega_{1}: \cdots: \omega_{p}\right]=-\left(\omega_{1}-\omega_{2}\right) \wedge \Delta\left[\omega_{2}: \cdots: \omega_{p}\right]$.

(4) $\Delta\left[\omega_{1}: \cdots: \omega_{p}\right]=(-1)^{p-1}\left(\omega_{1}-\omega_{2}\right) \wedge\left(\omega_{2}-\omega_{3}\right) \wedge \cdots \wedge\left(\omega_{p-1}-\omega_{p}\right)$.

(5) $\Delta\left[\omega_{1}: \cdots: \omega_{p}\right]=\left(\omega_{2}-\omega_{1}\right) \wedge\left(\omega_{3}-\omega_{1}\right) \wedge \cdots \wedge\left(\omega_{p}-\omega_{1}\right)$.

(6) $\omega_{1} \wedge \Delta\left[\omega_{1}: \cdots: \omega_{p}\right]=\omega_{1} \wedge \omega_{2} \wedge \cdots \wedge \omega_{p}$.

By (6), if $\Delta\left[\omega_{1}: \cdots: \omega_{p}\right]=0$, then $\omega_{1}, \ldots, \omega_{p}$ are $F$-linearly dependent. However, the converse is not true in general. The rational function $f \in F$ is said to be homogeneous of degree $d$, if $f=g_{1} / g_{2}$ with homogeneous polynomials $g_{1}, g_{2}$ and $d=\operatorname{deg} g_{1}-\operatorname{deg} g_{2}$.

Lemma 3. Assume $p \geq 2$ and $f_{1}, \ldots, f_{p} \in F \backslash K$ are homogeneous of the same degree. Then $\Delta\left[\mathrm{d} f_{1} / f_{1}: \cdots: \mathrm{d} f_{p} / f_{p}\right]=0$, if and only if, $\mathrm{d} f_{1} / f_{1} \wedge \cdots \wedge \mathrm{d} f_{p} / f_{p}=0$.

Proof. Let $\omega_{1}, \ldots, \omega_{p} \in \Omega^{1}(V)$.

From Lemma 2 we obtain:

(1) $\Delta\left[\omega_{1}: \cdots: \omega_{p}\right]=0$ if and only if there exists $\left(g_{1}, \ldots, g_{p}\right) \in F^{p} \backslash\{(0, \ldots, 0)\}$ such that $g_{1}+\cdots+g_{p}=0$ and $g_{1} \omega_{1}+\cdots+g_{p} \omega_{p}=0$.

(2) Take $\omega_{i}=\mathrm{d} f_{i} / f_{i}$ and $g_{i}=h_{i} f_{i}$. For $p \geq 2$ and $f_{1}, \ldots, f_{p} \in F \backslash K$, we have $\Delta\left[\mathrm{d} f_{1} / f_{1}: \cdots: \mathrm{d} f_{p} / f_{p}\right]=0$ if and only if there exists $\left(h_{1}, \ldots, h_{p}\right) \in$ $F^{p} \backslash\{(0, \ldots, 0)\}$ such that $h_{1} f_{1}+\cdots+h_{p} f_{p}=0$ and $h_{1} \mathrm{~d} f_{1}+\cdots+h_{p} \mathrm{~d} f_{p}=0$.

When the $f_{i}$ 's are homogeneous of the same degree, we can use the Euler derivation to conclude that $h_{1} \mathrm{~d} f_{1}+\cdots+h_{p} \mathrm{~d} f_{p}=0$ implies $h_{1} f_{1}+\cdots+h_{p} f_{p}=0$.

In particular, if $c_{1} f_{1}+\cdots+c_{p} f_{p}=0$ for some $\left(c_{1}, \ldots, c_{p}\right) \in K^{p} \backslash\{(0, \ldots, 0)\}$, then $\Delta\left[\mathrm{d} f_{1} / f_{1}: \cdots: \mathrm{d} f_{p} / f_{p}\right]=0$. If $f_{1}^{n_{1}} \cdots f_{p}^{n_{p}}=1$ for some non-zero integers $n_{1}, \ldots, n_{p}$, then $\Delta\left[\mathrm{d} f_{1} / f_{1}: \cdots: \mathrm{d} f_{p} / f_{p}\right]=0$. 
Remark. The derivation $\Delta$ is a natural generalization of the linear derivation on Orlik-Solomon Algebras ([OT]) in the degree one case.

\section{Proofs}

Proof of Theorem 1. Let $\left[x_{0}: x_{1}: \ldots: x_{n}\right]$ be homogeneous coordinates of $\mathbb{P}^{n}$. We can assume $D_{i}$ is given by a homogeneous polynomial $F_{i}(x)$ of degree $d$. So we can write $\omega_{\lambda}=\sum_{i=1}^{m} \lambda_{i} \mathrm{~d} F_{i} / F_{i}$. It is easy to check that $\mathrm{d} F_{i} / F_{i}-\mathrm{d} F_{j} / F_{j}$ and $\omega_{\lambda}$ are global forms. For $1 \leq i_{1}, \ldots, i_{p} \leq m$, define a holomorphic form on $M$ by

$$
\eta\left[i_{1}, \ldots, i_{p}\right]:=\Delta\left[\frac{\mathrm{d} F_{i_{1}}}{F_{i_{1}}}: \cdots: \frac{\mathrm{d} F_{i_{p}}}{F_{i_{p}}}\right] .
$$

According to Lemma 2 (4), if it is not zero, then $\eta\left[i_{1}, \ldots, i_{p}\right]$ is a global $(p-1)$-form. By (A1) and (A3), $F_{1}, \ldots, F_{n}$ becomes a basis of the vector subspace of $\Gamma\left(\mathbb{P}^{n}, \mathcal{O}(d)\right)$ defining the linear system $\Lambda$. So we can write $F_{j}=a_{1 j} F_{1}+\cdots+a_{n j} F_{n}$ for some constants $a_{i j}$. Define the $n \times s$-matrix $A=\left(a_{i j}\right)$. By (A3), any $n \times n$-minor of $A$ is not zero. Due to Lemma 3, we have $\eta\left[i_{1}, \ldots, i_{n+1}\right]=0$ for $1 \leq i_{1}<\cdots<i_{n+1} \leq s$. Because we can write $\omega_{\lambda}=\sum_{i \neq i_{1}} \lambda_{i}\left(\mathrm{~d} F_{i} / F_{i}-\mathrm{d} F_{i_{1}} / F_{i_{1}}\right)$, using Lemma 3 we have $\omega_{\lambda} \wedge \eta\left[i_{1}, \ldots, i_{n}\right]=0$. Hence $\nabla_{\lambda}\left(\eta\left[i_{1}, \ldots, i_{n}\right]\right)=0$ for $1 \leq i_{1}<\cdots<i_{n} \leq s$. We note that (A2) and (A3) imply that for $1 \leq i_{1}<\cdots<i_{n} \leq s$, we have $\mathrm{d} F_{i_{1}} / F_{i_{1}} \wedge \cdots \wedge \mathrm{d} F_{i_{n}} / F_{i_{n}} \neq 0$, and by Lemma $\left\{, \eta\left[i_{1}, \ldots, i_{n}\right] \neq 0\right.$. Thus $\eta\left[i_{1}, \ldots, i_{n}\right]$ is a $\nabla_{\lambda}$-closed $(n-1)$-form for $1 \leq i_{1}<\cdots<i_{n} \leq s$.

By (A2), we may take a local neighborhood $U$ and coordinates $x=\left(x_{1}, \ldots, x_{n}\right)$ at $P$ such that $D_{i}$ is defined by $x_{i}=0$ for $i=1, \ldots, n$. Let $\alpha_{j}(x)=a_{1 j} x_{1}+\cdots+$ $a_{n j} x_{n}$ and $H_{j}=\left\{\alpha_{j}(x)=0\right\}$. So we get the central arrangement $\mathcal{A}=\left\{H_{j}\right\}_{1 \leq j \leq s}$ of hyperplanes in $\mathbb{C}^{n} \simeq U\left(\bigcap_{i=1}^{s} H_{i}\right.$ is the origin). Let $M(\mathcal{A})$ denote the complement of the arrangement $\mathcal{A}$. Then we have $H^{k}\left(U \cap M,\left.\mathcal{L}_{\lambda}\right|_{U \cap M}\right)=H^{k}\left(M(\mathcal{A}), \tilde{\mathcal{L}}_{\lambda}\right)$, where $\tilde{\mathcal{L}}_{\lambda}$ is the rank one local system on $M(\mathcal{A})$ whose monodromy around the hyperplane $H_{j}$ is $\exp \left(-2 \pi \sqrt{-1} \lambda_{j}\right)$. Since $\lambda$ is non-trivial and $\sum_{i=1}^{s} \lambda_{i}=0$, without loss of generality we may assume that $\lambda_{1}$ and $\lambda_{s}$ are not integers. Now, choosing $H_{1} \in \mathcal{A}$, we get the decone $\mathbf{d} \mathcal{A}$ (see [OT]), which is an arrangement of $s-1$ affine hyperplanes in $\mathbb{C}^{n-1} \simeq H_{1}$. Note that $M(\mathcal{A}) \simeq M(\mathbf{d} \mathcal{A}) \times \mathbb{C}^{*}$ by the restriction of the Hopf bundle. Since any $n \times n$-minor of $A$ is not zero, $\mathcal{A}$ is generic and $\mathbf{d} \mathcal{A}$ is in general position ( $\mathrm{OT})$. On the other hand, for the integer weight $k \in \mathbb{Z}^{n}$ with $\sum_{i=1}^{s} k_{i}=0$, we know that the local system $\tilde{\mathcal{L}}_{\lambda}$ is equivalent to the local system $\tilde{\mathcal{L}}_{\lambda+k}$ associated to the integer shift weight $\lambda+k$ (see OT2]). By shifting a weight if necessary, we can assume that $\lambda \notin(\mathbb{Z} \backslash\{0\})^{n}$. Since $H^{k}\left(M(\mathcal{A}), \tilde{\mathcal{L}}_{\lambda}\right) \simeq$ $H^{k}\left(M(\mathbf{d} \mathcal{A}), \tilde{\mathcal{L}}_{\lambda}\right) \oplus H^{k-1}\left(M(\mathbf{d} \mathcal{A}), \tilde{\mathcal{L}}_{\lambda}\right)($ cf. $[\mathrm{Fa}])$, in this case the following is known.

Lemma 4 (cf. [Ha, $\mathrm{KN}$, Ki, $\mathrm{Ka}$ ). Let $\mathcal{A}=\left\{H_{j}=\left\{\alpha_{j}=0\right\}: 1 \leq j \leq s\right\}$ be a generic arrangement of hyperplanes in $\mathbb{C}^{n}$ and let $M(\mathcal{A})$ be its complement. For a complex weight $\lambda=\left(\lambda_{1}, \ldots, \lambda_{s}\right)$ such that $\lambda_{1} \notin \mathbb{Z}, \lambda_{s} \notin \mathbb{Z}$ and $\sum_{i=1}^{s} \lambda_{i}=0$, we have

(1) $H^{k}\left(M(\mathcal{A}), \tilde{\mathcal{L}}_{\lambda}\right)=0$ for $k \neq n, n-1$,

(2) $H^{n}\left(M(\mathcal{A}), \tilde{\mathcal{L}}_{\lambda}\right) \simeq H^{n-1}\left(M(\mathcal{A}), \tilde{\mathcal{L}}_{\lambda}\right)$ and $\operatorname{dim} H^{n}=\operatorname{dim} H^{n-1}=\left(\begin{array}{l}s-2 \\ n-1\end{array}\right)$,

(3) $\left\{e_{1} \wedge e_{i_{1}} \wedge \cdots \wedge e_{i_{n-1}}: 1<i_{1}<\cdots<i_{n-1}<s\right\}$ is a basis of $H^{n}$,

(4) $\left\{\Delta\left[e_{1}: e_{i_{1}}: \cdots: e_{i_{n-1}}\right]: 1<i_{1}<\cdots<i_{n-1}<s\right\}$ is a basis of $H^{n-1}$,

where $e_{j}=\mathrm{d} \alpha_{j} / \alpha_{j}$ and $\tilde{\mathcal{L}}_{\lambda}$ is the rank one local system on $M(\mathcal{A})$ whose monodromy around the hyperplane $H_{j}$ is $\exp \left(-2 \pi \sqrt{-1} \lambda_{j}\right)$. 
Note that $H^{k}\left(M(\mathcal{A}), \tilde{\mathcal{L}}_{\lambda}\right)$ is isomorphic to the twisted de Rham cohomology defined by the one form $e_{\lambda}=\sum_{j=1}^{s} \lambda_{j} e_{j}$ (see [OT2]) and that $\left.\omega_{\lambda}\right|_{U}=e_{\lambda}$ and $\left.\eta\left[i_{1}, \ldots, i_{n}\right]\right|_{U}=\Delta\left[e_{i_{1}}: \cdots: e_{i_{n}}\right]$. Now suppose that there exists a global $(n-$ 2 )-form $\alpha$ such that $\eta\left[i_{1}, \ldots, i_{n}\right]=\nabla_{\lambda}(\alpha)$. Then restricting it to $U$, we have $\Delta\left[e_{i_{1}}: \cdots: e_{i_{n}}\right]=\tilde{\nabla}_{\lambda}\left(\left.\alpha\right|_{U}\right)$ where $\tilde{\nabla}_{\lambda}=d+e_{\lambda} \bigwedge$. However, by the above lemma, this is a contradiction. Therefore $\eta\left[i_{1}, \ldots, i_{n}\right]$ defines a non-vanishing class of degree $n-1$ for $1 \leq i_{1}<\cdots<i_{n} \leq s$. In a similar fashion, we obtain $\left\{\eta\left[1, i_{1}, \ldots, i_{n-1}\right]\right.$ : $\left.1<i_{1}<\cdots<i_{n-1}<s\right\}$ as a linearly independent set of elements of $H^{n-1}\left(M, \mathcal{L}_{\lambda}\right)$.

Assume $s<m$ and fix $m$. Take $\eta\left[m, i_{1}, \ldots, i_{n}\right]$ for $1 \leq i_{1}<\cdots<i_{n} \leq s$. It is easy to see that $\left.\eta\left[m, i_{1}, \ldots, i_{n}\right]\right|_{U}=e_{i_{1}} \wedge \cdots \wedge e_{i_{n}}$. Therefore, $\eta\left[m, i_{1}, \ldots, i_{n}\right]$ defines a non-vanishing class of degree $n$. Similary, using the above lemma, we have $\left\{\eta\left[m, 1, i_{1}, \ldots, i_{n-1}\right]: 1<i_{1}<\cdots<i_{n-1}<s\right\}$ as a linearly independent set of elements of $H^{n}\left(M, \mathcal{L}_{\lambda}\right)$. This completes the proof.

Corollary 5. Under the assumptions of Theorem 1, suppose $s=m$ and that $D_{1}+\cdots+D_{s}$ has an irreducible component not passing through fixed P. Namely, at least one of $D_{1}, \ldots, D_{s}$ is reduced and it has an irreducible component not passing through $P$. For example, $D_{1}, \ldots, D_{s}$ are divisors of degree $d>1$ defined only by hyperplanes such that the arrangement of their hyperplanes is not central. Then we have

$$
\operatorname{dim} H^{n-1}\left(M, \mathcal{L}_{\lambda}\right) \geq\left(\begin{array}{l}
s-2 \\
n-1
\end{array}\right) \text { and } \operatorname{dim} H^{n}\left(M, \mathcal{L}_{\lambda}\right) \geq\left(\begin{array}{l}
s-2 \\
n-1
\end{array}\right) .
$$

Proof. We need to prove the latter inequality. Let $D^{\prime}$ be one of irreducible components of $D_{1}+\cdots+D_{s}$ not passing through $P$ with degree $d^{\prime}<d$. We can modify the last part of the proof of Theorem 1 as follows. Let $F^{\prime}$ be a defining homogeneous polynomial of $D^{\prime}$. The argument used in the proof of Theorem 1 shows that

$$
\Delta\left[\left(d / d^{\prime}\right) \frac{\mathrm{d} F^{\prime}}{F^{\prime}}: \frac{\mathrm{d} F_{1}}{F_{1}}: \frac{\mathrm{d} F_{i_{1}}}{F_{i_{1}}}: \cdots: \frac{\mathrm{d} F_{i_{n-1}}}{F_{i_{n-1}}}\right]: 1<i_{1}<\cdots<i_{n-1}<s
$$

is independent in $H^{n}\left(M, \mathcal{L}_{\lambda}\right)$.

If a weight $\lambda$ is trivial, then $H^{k}\left(M, \mathcal{L}_{\lambda}\right)$ is isomorphic to the usual de Rham cohomology $H^{k}(M)$ on $M$.

Corollary 6. Under the assumption of Theorem 1, we have

$$
\operatorname{dim} H^{k}(M) \geq\left(\begin{array}{c}
s-1 \\
k
\end{array}\right) \quad \text { for } 1 \leq k \leq n-1 .
$$

Moreover, if $s<m$, then we have

$$
\operatorname{dim} H^{n}(M) \geq\left(\begin{array}{l}
s-1 \\
n-1
\end{array}\right) \text { and } \operatorname{dim} H^{k}(M) \geq\left(\begin{array}{l}
s \\
k
\end{array}\right) \text { for } 1 \leq k \leq n-1 .
$$

Proof. In the proof of Theorem 1, since $\mathcal{A}$ is the generic arrangement of $s$ hyperplanes in $\mathbb{C}^{n}$ and its decone $\mathbf{d} \mathcal{A}$ is in general position, the following is known ( $\underline{\mathrm{OT}})$ :

(1) $\operatorname{dim} H^{k}(M(\mathcal{A}))=\left(\begin{array}{l}s \\ k\end{array}\right)$ for $1 \leq k \leq n$ and $\operatorname{dim} H^{k}(M(\mathbf{d} \mathcal{A}))=\left(\begin{array}{c}s-1 \\ k\end{array}\right)$ for $1 \leq k \leq n-1$

(2) $H^{k}(M(\mathcal{A})) \simeq H^{k}(M(\mathbf{d} \mathcal{A})) \oplus H^{k-1}(M(\mathbf{d} \mathcal{A}))$ for $1 \leq k \leq n-1$, and $H^{n}(M(\mathcal{A})) \simeq H^{n-1}(M(\mathbf{d} \mathcal{A}))$

(3) $\left\{e_{1} \wedge e_{i_{1}} \wedge \cdots \wedge e_{i_{n-1}}: 1<i_{1}<\cdots<i_{n-1} \leq s\right\}$ is a basis of $H^{n}(M(\mathcal{A}))$, 
(4) $\left\{\Delta\left[e_{1}: e_{i_{1}}: \cdots: e_{i_{k}}\right]: 1<i_{1}<\cdots<i_{k} \leq s\right\} \cup\left\{e_{1} \wedge e_{i_{1}} \wedge \cdots \wedge e_{i_{k-1}}: 1<\right.$ $\left.i_{1}<\cdots<i_{k-1} \leq s\right\}$ is a basis of $H^{k}(M(\mathcal{A}))$ for $1 \leq k \leq n-1$.

Since $\eta\left[i_{1}, \ldots, i_{k}\right]$ is $d$-closed, the same argument proves this corollary.

\section{Generalization}

Let $\mathcal{A}$ be an arrangement of (affine or projective) hyperplanes. The intersection set $L(\mathcal{A})$ of $\mathcal{A}$ is the set of non-empty intersections of elements of $\mathcal{A}$. For $X \in L(\mathcal{A})$, define a central arrangement $\mathcal{A}_{X}=\{H \in \mathcal{A} \mid X \subset H\}$. Let $\mathcal{C}$ be a central arrangement with center $\bigcap_{H \in \mathcal{C}} H \neq \emptyset$. We call $\mathcal{C}$ decomposable if there exist nonempty subarrangements $\mathcal{C}_{1}$ and $\mathcal{C}_{2}$ so that $\mathcal{C}=\mathcal{C}_{1} \cup \mathcal{C}_{2}$ is a disjoint union, and after a linear coordinate change, the defining polynomials for $\mathcal{C}_{1}$ and $\mathcal{C}_{2}$ have no common variables. Define $\mathrm{D}(\mathcal{A})=\left\{X \in L(\mathcal{A}): \mathcal{A}_{X}\right.$ is not decomposable $\}$. For a complex weight $\lambda$ of $\mathcal{A}$ and $X \in L(\mathcal{A})$, denote $\lambda_{X}=\sum_{H \in \mathcal{A}_{X}} \lambda_{H}$. The construction of a basis for the twisted cohomology for arrangements given in [FT] (cf. OT2]), together with the arguments used in proof of Theorem 1, shows the following.

Theorem 7. Let $1<n<s \leq m$. Let $D_{1}, \ldots, D_{m}$ be effective divisors on $\mathbb{P}^{n}$ with the same degree and let $M$ be the complement of their supports. Suppose (A1) and (A2) hold. Let $\mathcal{A}$ be the arrangement of hyperplanes in the dual projective space $\Lambda^{*}=\left(\mathbb{P}^{n-1}\right)^{*} \simeq \mathbb{P}^{n-1}$ defined by $D_{1}, \ldots, D_{s}$. Let $\lambda$ be a non-trivial weight such that $\sum_{i=1}^{s} \lambda_{i}=0$ and $\lambda_{i}=0$ for $i=s+1, \ldots, m$. If $\lambda_{X} \notin \mathbb{Z}_{\geq 0}$ for every $X \in \mathrm{D}(\mathcal{A})$, then we have

$$
\operatorname{dim} H^{n-1}\left(M, \mathcal{L}_{\lambda}\right) \geq \beta,
$$

and moreover, if $s<m$, then we have

$$
\operatorname{dim} H^{n}\left(M, \mathcal{L}_{\lambda}\right) \geq \beta,
$$

where $\beta$ is the Euler characteristic $\chi(M(\mathcal{A}))$ of $M(\mathcal{A})=\mathbb{P}^{n-1} \backslash \bigcup_{H \in \mathcal{A}} H$.

Remark. Note that $\beta$ is known as the beta invariant of the underlying matroid of $\mathcal{A}$. If $\mathcal{A}$ is defined over real, then $\beta$ is the number of bounded chambers in $\mathbb{R}^{n-1}=\mathbb{P}^{n-1}(\mathbb{R}) \backslash H$ for $H \in \mathcal{A}$ (see [STV], OT2]).

Remark. One has $\lambda_{H} \notin \mathbb{Z}_{\geq 0}$ for all hyperplanes in $\mathcal{A}$. We can generalize this theorem to the case in which there is a hyperplane $H \in \mathcal{A}$ with $\lambda_{H}=0$ by using Ka2.

\section{AfFine CASE}

Let $V_{1}^{a}, \ldots, V_{m}^{a}$ be hypersurfaces in the complex affine space $\mathbb{C}^{n}$ with coordinates $u=\left(u_{1}, \ldots, u_{n}\right)$. Write $V^{a}=\bigcup_{i=1}^{m} V_{i}^{a}$ and $M=\mathbb{C}^{n} \backslash V^{a}$. Assume that $V_{j}^{a}$ is defined by a polynomial $f_{j}(u)$ of degree $d_{i}$. Let $\lambda=\left(\lambda_{1}, \ldots, \lambda_{m}\right)$ be a weight and let $\omega_{\lambda}^{a}=\sum_{i=1}^{m} \lambda_{i} \mathrm{~d} f_{i} / f_{i}$. Then we obtain the twisted de Rham complex $\left(\Omega\left(* V^{a}\right), \nabla_{\lambda}^{a}\right)$ where $\Omega\left(* V^{a}\right)$ is the space of rational forms with poles along $V^{a}$ and $\nabla_{\lambda}^{a}=d+\omega_{\lambda}^{a} \wedge$. The Grothendiek-Deligne comparison theorem ([De] $)$ asserts that

$$
H^{k}\left(M, \mathcal{L}_{\lambda}^{a}\right) \simeq H^{k}\left(\Omega\left(* V^{a}\right), \nabla_{\lambda}^{a}\right)
$$

where $\mathcal{L}_{\lambda}^{a}$ is the rank one local system defined by the flat connection $\nabla_{\lambda}^{a}$ (cf. $[\mathrm{KN}$, Ki ).

View $\mathbb{P}^{n}$ as a compactification of $\mathbb{C}^{n}$ with hyperplane $H_{\infty}$ at infinity. Let $\left[x_{0}\right.$ : $\left.\ldots: x_{n}\right]$ denote homogeneous coordinates with $H_{\infty}=\left\{x_{0}=0\right\}$. Define the homogeneous polynomial $F_{j}(x)=x_{0}^{d} f_{j}\left(x_{1} / x_{0}, \ldots, x_{n} / x_{0}\right)$ of degree $d=\max \left(d_{1}, \ldots, d_{m}\right)$. 
Then $F_{j}(x)$ determines the divisor $D_{j}$ of degree $d$. If $d=d_{1}=\cdots=d_{m}$, then the support of $\sum_{i=1}^{m} D_{i}$ does not contain $H_{\infty}$; otherwise it contains $H_{\infty}$. Note that the weight of $H_{\infty}$ is given by $-\sum_{i=1}^{m} \lambda_{i} d_{j}$. If $d=d_{1}=\cdots=d_{m}$ and $\sum_{i=1}^{m} \lambda_{i}=0$, then the weight of $H_{\infty}$ is zero.

Corollary 8. Under the assumptions of Theorem 1, if a point $P$ in (A2) is not on $H_{\infty}$, then we have

$$
\operatorname{dim} H^{n-1}\left(M^{a}, \mathcal{L}_{\lambda}^{a}\right) \geq\left(\begin{array}{c}
s-2 \\
n-1
\end{array}\right)
$$

for a non-trivial weight $\lambda$ with $\sum_{i=1}^{s} \lambda_{i}=0$ and $\lambda_{i}=0$ for $i=s+1, \ldots, m$.

\section{EXAMPLES}

6.1. $n=2$ and $s=3$. Let $D_{1}, D_{2}$ and $D_{3}$ be irreducible prime divisors on $\mathbb{P}^{2}$ defined by $F_{1}=x_{0}^{2}+x_{1}^{2}-2 x_{2}^{2}, F_{2}=x_{0}^{2}+2 x_{1}^{2}-3 x_{2}^{2}$ and $F_{3}=2 x_{0}^{2}+x_{1}^{2}-3 x_{2}^{2}$, respectively. They are three generic elements of the pencil of conic curves $F_{[a: b]}=$ $a\left(x_{0}^{2}-x_{2}^{2}\right)+b\left(x_{1}^{2}-x_{2}^{2}\right)$ and they transversally intersect each other at each of the four intersection points. Let $M=\mathbb{P}^{2} \backslash \bigcup_{i=1}^{3} D_{i}$ and $M^{a}=\mathbb{C}^{2} \backslash \bigcup_{i=1}^{3}\left(D_{i} \cap \mathbb{C}^{2}\right)$ where $\mathbb{C}^{2}=\mathbb{P}^{2} \backslash\left\{x_{2}=0\right\}$. We have $H^{1}\left(M, \mathcal{L}_{\lambda}\right) \neq 0$ and $H^{1}\left(M^{a}, \mathcal{L}_{\lambda}\right) \neq 0$ for a non-trivial weight $\lambda$ with $\lambda_{1}+\lambda_{2}+\lambda_{3}=0$. In the following cases, we can also arrive at this conclusion:

(1) $F_{1}=x_{0}^{2}+x_{1}^{2}-2 x_{2}^{2}, F_{2}=x_{0}^{2}+2 x_{1}^{2}-3 x_{2}^{2}$ and $F_{3}^{\prime}=x_{0}^{2}-x_{1}^{2}$ (two conics and a set of 2-lines).

(2) $F_{1}=x_{0}^{2}+x_{1}^{2}-2 x_{2}^{2}, F_{2}^{\prime}=x_{2}^{2}-x_{0}^{2}$ and $F_{3}^{\prime}=x_{0}^{2}-x_{1}^{2}$ (one conic and two sets of 2-lines).

(3) $F_{1}^{\prime}=x_{1}^{2}-x_{2}^{2}, F_{2}^{\prime}=x_{2}^{2}-x_{0}^{2}$ and $F_{3}^{\prime}=x_{0}^{2}-x_{1}^{2}$ (three sets of 2-lines).

In the last case, one has an arrangement of 6 lines as in the Ceva Theorem. In a similar fashion, we get the following. In Pascal's theorem, define divisors $D_{1}, D_{2}$ and $D_{3}$ with degree three by one conic and one pascal line, three lines, and another three lines, respectively. The resulting complement has a non-vanishing twisted cohomology. Needless to say, the same is true for the complement of 9 lines in Pappus's theorem $([\mathrm{Fa}])$. In the degree four case, there are two different arrangements of 12 lines in the Kirkman Theorem and the Steiner Theorem ([Ka3]). Those arrangements are 3-nets, whose combinatorial structures are matroids associated to Latin squares ([LY, $\mathrm{Yu} 2, \mathrm{Ka} 3]$ ).

On the other hand, the $B_{3}$-arrangement is an example of the case that $D_{i}$ 's are not prime. Let $D_{1}, D_{2}$ and $D_{3}$ be divisors defined by $x_{2}^{2}\left(x_{0}^{2}-x_{1}^{2}\right), x_{1}^{2}\left(x_{0}^{2}-x_{2}^{2}\right)$ and $x_{0}^{2}\left(x_{1}^{2}-x_{2}^{2}\right)$, respectively. Their divisors can be written by $D_{1}=2 H_{1}+H_{2}+H_{3}$, $D_{2}=2 H_{4}+H_{5}+H_{6}$ and $D_{3}=2 H_{7}+H_{8}+H_{9}$, where $H_{i}$ 's are hyperplanes. The arrangement $\mathcal{A}=\left\{H_{1}, \ldots, H_{9}\right\}$ is called the $B_{3}$-arrangement. Note that a weight $\lambda$ induces the weight $\left(2 \lambda_{1}, \lambda_{1}, \lambda_{1}, 2 \lambda_{2}, \lambda_{2}, \lambda_{2}, 2 \lambda_{3}, \lambda_{3}, \lambda_{3}\right)$ of $\mathcal{A}$ (cf. [Fa, Ka3]).

Consider the divisors defined by $\left(x_{0}-x_{2}\right)\left(x_{0}+x_{2}\right), x_{2}\left(x_{2}-x_{1}\right)$ and $x_{0}^{2}-x_{1} x_{2}$. They are tangent at $[0: 1: 0]$. However, by looking locally at $[1: 1: 1]$ and $[-1: 1: 1]$, condition (A2) holds and thus the complement of the union of these divisors has a non-vanishing twisted cohomology.

We can make an example with a singular curve. Let $F_{1}=x_{2}^{3}-x_{1}^{2} x_{0}, F_{2}=$ $x_{3}^{2}\left(x_{0}-x_{3}\right)$ and $F_{3}=x_{0}\left(x_{1}-x_{2}\right)\left(x_{1}+x_{2}\right)$. The curve $F_{1}=0$ has a cusp singularity, and the resulting complement has a non-vanishing twisted cohomology. 
6.2. $n=2$ and $s \geq 3$. We consider the pencil of cubic curves

$$
F_{[a: b]}=a\left(x_{0}^{3}+x_{1}^{3}+x_{2}^{3}\right)+3 b x_{0} x_{1} x_{2} .
$$

A generic element given by $a \neq 0$ and $b^{3} \neq-1$ is non-singular. For $s$ generic elements $D_{1}, \ldots, D_{s}$, we have $\operatorname{dim} H^{1}\left(M, \mathcal{L}_{\lambda}\right) \geq s-2$. Define non-generic elements $F_{1}=x_{0} x_{1} x_{2}, F_{2}=x_{0}^{3}+x_{1}^{3}+x_{2}^{3}-3 x_{0} x_{1} x_{2}, F_{3}=x_{0}^{3}+x_{1}^{3}+x_{2}^{3}-3 \xi x_{0} x_{1} x_{2}$ and $F_{4}=x_{0}^{3}+x_{1}^{3}+x_{2}^{3}-3 \xi^{2} x_{0} x_{1} x_{2}$ where $\xi=e^{2 \pi \sqrt{-1} / 3}$. They are four sets of 3-lines, and the set $\mathcal{A}$ of all lines is called the Hessian configuration, which is the arrangement of 12 lines passing through the nine inflection points of a non-singular cubic. In this case, we know $\operatorname{dim} H^{1}\left(M, \mathcal{L}_{\lambda}\right)=2$ for a non-trivial weight $\lambda$ with $\sum_{i=1}^{4} \lambda_{i}=0$ ( $\mathrm{Li}]$ ).

6.3. $n=3$. Let

$$
\begin{gathered}
F_{1}=x_{0}\left(x_{1}+x_{2}+x_{3}\right), \\
F_{2}=x_{1}\left(-x_{0}+x_{2}-x_{3}\right), \\
F_{3}=x_{2}\left(-x_{0}-x_{1}+x_{3}\right)
\end{gathered}
$$

and

$$
F_{4}=x_{3}\left(-x_{0}+x_{1}-x_{2}\right) \text {. }
$$

One can verify that the divisors defined by them satisfy the conditions in Theorem 1 . Then $H^{2}\left(M, \mathcal{L}_{\lambda}\right) \neq 0$ for a weight $\lambda$ with $\sum_{i=1}^{4} \lambda_{i}=0$. This yields an arrangement of 8 planes whose underlying matroid is of type $L_{8}$ ([Ka3]).

6.4. A higher dimensional case. Let $F_{i}=x_{i-1}^{d}-x_{i}^{d}$ for $i=1, \ldots, n$ and $F_{0}=x_{n}^{d}-x_{0}^{d}$. The support of the associated divisors determines an arrangement $\mathcal{A}$ of $(n+1) d$ hyperplanes in $\mathbb{P}^{n}$. Note that this is a projective closure of a subarrangement of the monomial arrangement $\mathcal{A}_{d, d, n+1}$ (see [OT, [CS]). Since $\sum_{k=0}^{n} F_{k}=0$, we have $H^{n-1}\left(M, \mathcal{L}_{\lambda}\right) \neq 0$ for a weight $\lambda$ with $\sum_{i=0}^{n} \lambda_{i}=0$. The $n=2$ case was found in [CS. Note that the underlying matroid of $\mathcal{A}$ is a degeneration of the matroid associated to the Latin $n$-dimensional hypercube given by the addition table for $\left(\mathbb{Z}_{d}\right)^{n}$ (see $[\mathrm{Ka} 3]$ ).

\section{REFERENCES}

[A] K. Aomoto, Un théorème du type de Matsushima-Murakami concernant l'intégrale des fonctions multiformes, J. Math. Pures Appl. 52 (1973), 1-11. MR0396563 (53:426)

[Ch] K. Cho, A generalization of Kita and Noumi's vanishing theorems of cohomology groups of local system, Nagoya Math. J. 147 (1997), 63-69. MR1475166 (99a:14025)

[CDO] D. C. Cohen, A. Dimca, and P. Orlik, Nonresonance conditions for arrangements, Ann. Inst. Fourier 53 (2003), no. 6, 1883-1896. MR2038782 (2005a:32027)

[CS] D. Cohen, and A. Suciu, Characteristic varieties of arrangements, Math. Proc. Cambridge Philos. Soc. 127 (1999), 33-54. MR1692519 (2000m:32036)

[De] P. Deligne, Équations différentielles à points singuliers réguliers, Lecture Notes in Math. 163 (1970). MR0417174 (54:5232)

[Di] A. Dimca, Sheaves in topology, Universitext. Springer-Verlag, Berlin, 2004. xvi+236 pp. MR2050072 (2005j:55002)

[ESV] H. Esnault, V. Schechtman and E. Viehweg, Cohomology of local systems on the complement of hyperplanes, Invent. Math. 109 (1992), 557-561; Erratum 112 (1993), 447. MR 1176205 (93g:32051)

[Fa] M. Falk, Arrangements and Cohomology, Annals of Comb. 1 (1997), 135-157. MR1629681 (99g:52017)

[FT] M. Falk and H. Terao, $\beta$ NBC-bases for cohomology of local systems on hyperplane complements, Trans. Amer. Math. Soc. 349 (1997), no. 1, 189-202. MR1401770 (97g:52029) 
[H] R. Hartshorne, Algebraic Geometry, GTM 52, Springer, 1977. MR0463157 (57:3116)

[Ha] A. Hattori, Topology of $\mathbb{C}^{n}$ minus a finite number of affine hyperplanes in general position, J. Fac. Sci. Univ. Tokyo, Sect. IA 22 (1975), 205-219. MR0379883 (52:788)

[Ka] Y. Kawahara, The twisted De Rham cohomology for Basic Constructions of Hyperplane arrangements and its applications, Hokkaido Mathematical Journal 34 (2005), no. 2, 489505. MR2159008 (2006e:14021)

[Ka2] Y. Kawahara, Vanishing and bases for cohomology of partially trivial local systems on hyperplane arrangements, Proceedings of the American Mathematical Society 133 (2005), 1907-1915. MR2137854 (2007b:32044)

[Ka3] Y. Kawahara, The non-vanishing cohomology of Orlik-Solomon algebras, Tokyo Journal of Mathematics, 30 (2007), no. 1, 223-238. MR.2328065

[Ki] M. Kita, On vanishing of the twisted rational de Rham cohomology associated with hypergeometric functions, Nagoya Math. J. 135 (1994), 55-85. MR1295817 (95j:33043)

[KN] M. Kita and M. Noumi, On the structure of cohomology groups attached to the integral of certain many-valued analytic functions, Japan. J. Math. 9 (1983), no. 1, 113-157. MR722538 (84m:32040)

[Ko] T. Kohno, Homology of a local system on the complement of hyperplanes, Proc. Japan Acad. Ser. A 62 (1986), 144-147. MR846350 (87i:32019)

[Li] A. Libgober, Characteristic varieties of algebraic curves, Applications of algebraic geometry to coding theory, physics and computation (Eilat, 2001), 215-254, NATO Sci. Ser. II Math. Phys. Chem., 36, Kluwer Acad. Publ., Dordrecht, 2001. MR1866902 (2003e:14008)

[Li2] A. Libgober, Homotopy groups of complements to ample divisors, math.AG/0404341.

[LY] A. Libgober and S. Yuzvinsky, Cohomology of the Orlik-Solomon algebras and local systems, Compositio Math. 121 (2000), no. 3, 337-361. MR1761630 (2001j:52032)

[OT] P. Orlik, and H. Terao, Arrangements of Hyperplanes, Grundlehren der mathematischen Wissenschaften 300, Springer-Verlag, 1992. MR1217488 (94e:52014)

[OT2] P. Orlik, and H. Terao, Arrangements and Hypergeometric integrals, MSJ, Mem. vol. 9, Math. Soc. Japan (2001). MR.1814008 (2003a:32048)

[STV] V. Schechtman, H. Terao and A. Varchenko, Local systems over complements of hyperplanes and the Kac-Kazhdan conditions for singular vectors, J. Pure Appl. Algebra 100 (1995), 93-102. MR1344845 (96j:32047)

[Yu S. Yuzvinsky, Cohomology of the Brieskorn-Orlik-Solomon algebras, Comm. Algebra 23 (1995), 5339-5354. MR.1363606 (97a:52023)

[Yu2] S. Yuzvinsky, Realization of finite abelian groups by nets in $\mathbb{P}^{2}$. Compositio Math. 140 (2004), no. 6, 1614-1624. MR2098405 (2005g:52057)

Department of Mathematics, Tokyo Metropolitan University, Minami-Ohsawa 1-1, HACHIOJI-SHI, TOKYO 192-0397, JAPAN

E-mail address: kawahara@z2.keio.jp 